Published in final edited form as:

Macromolecules. 2018 September 11; 51(17): 6609-6622. doi:10.1021/acs.macromol.8b01052.

\title{
Statistical Damage Mechanics of Polymer Networks
}

\author{
Franck J. Vernerey ${ }^{\star}, \dagger, \neq$, Roberto Brighenti ${ }^{\S}$, Rong Long ${ }^{\dagger, \ddagger}$, Tong Shen ${ }^{\dagger}$ \\ † Department of Mechanical Engineering, University of Colorado Boulder, Boulder, Colorado \\ 80309, United States \\ ‡ Program of Materials Science and Engineering, University of Colorado Boulder, Boulder, \\ Colorado 80309, United States \\ $\S$ Department of Engineering \& Architecture, University of Parma, Parco Area delle Scienze \\ 181/A, 43124 Parma, Italy
}

\section{Abstract}

The macroscopic mechanical response of polymers can be traced down to the microscale physics of the network by using a statistical approach for the description of the configuration state of the polymer chains. In this paper we present a micromechanical model to capture the macroscopic behavior of polymers by tracking the evolution of a distribution function describing chain configurations, more specifically the statistics of the end-to-end distance on the network chains. Damage, manifested in the softening and hysteresis under cyclic loading, is accounted for through the scission of chains, whose occurrence is evaluated on the basis of the probability of failure, also settled in the configuration space. The proposed micromechanical model can easily accommodate also the mechanics of dynamic network with reversible cross-links, thereby providing a general and physics-based approach to the study of polymers and polymer-like materials.

\section{Graphic Abstract}
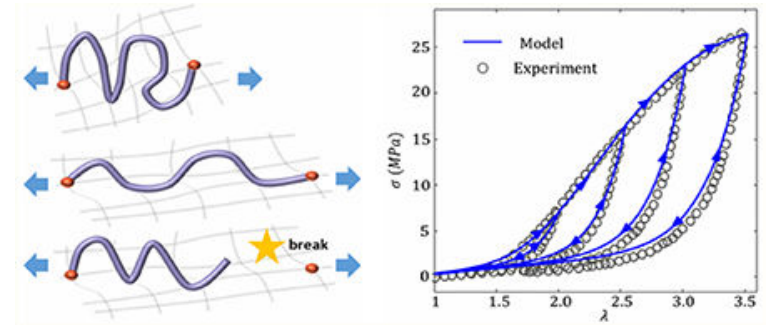

\section{INTRODUCTION}

Elastomers and gels are increasingly used in applications such as artificial muscles, tissue engineering, soft robotics, adhesives, and stretchable electronics. One reason these polymers are so desirable is because they can combine a myriad of functionalities with the ability to undergo very large deformations under fairly small forces. Despite these attractive

\footnotetext{
*Corresponding Author franck.vernerey@ colorado.edu (F.J.V.).
}

The authors declare no competing financial interest. 
properties, gels are often found to be brittle, which significantly limits their potential as structural materials (such as tissue scaffold). Although they can often undergo larger deformation, soft polymers are also limited by failure which may take the form of necking, fracture, or fatigue failure. These failure mechanisms may be better understood by taking a closer look at the molecular mechanisms at play. Elastomers and gels consisting of molecular networks of cross-linked chains are mainly governed by entropic elasticity. In other words, thermal fluctuations enable chains to explore a large configurational space in the absence of stretch. When chains are stretched, however, the number of configurations they can explore is reduced and the entropy of the network decreases. Statistical models have been successful at explaining the nonlinear elastic response of polymers based on this change of entropy. ${ }^{6,12,20,43}$ In the context of this work, we are particularly interested in the failure mechanisms that occur when individual chains are stretched beyond their load carrying capacity, i.e., their end-to-end distance become close to their contour length. In this case, entropic elasticity predicts a pronounced strain stiffening of the network, followed by a progressive failure of the constituent chains as they reach their critical rupture force, which leads to macroscale failure events such as necking or crack nucleation.

A number of strategies have been proposed to increase the toughness of soft polymers by increasing the dissipation mechanisms in the network during failure. One of them has been to create particle-reinforced polymer composites in which the process of particle debonding combined with polymer deformation significantly enhance dissipation. Increase in toughness, measured by the fracture energy, up to an order of magnitude ${ }^{24}$ was observed by this approach. Other approaches rely on the introduction of reversible bonds that can dynamically detach and re-form over time. This endows the material with a viscous component that can drastically enhance dissipative processes in the process zone of a crack. In this case, the toughness was found to be rate dependent and can increase by as much as 2 orders of magnitude, ${ }^{40}$ depending on the nature of the reversible bond. More recently, a number of groups have proposed the idea of combining multiple networks within the same polymer. ${ }^{13,14} \mathrm{~A}$ tight and brittle network can enhance the stiffness while acting as a sacrificial component during fracture. A second network, more loose and flexible, may then be used to bridge fractured regions and enhance energy dissipation yielded by the first network. This concept has proven extremely successful, most notably for the toughening of hydrogels which displayed a very large improvement in their fracture strain. More work is currently underway to explore the possibility of incorporating more than two networks.

With our increasing capacity to control molecular structures, the need of a better comprehension of the structure/toughness relationship is becoming more apparent. In this context, the development of mathematical models that can serve to guide the experimental design to optimize fracture resistance is highly desirable. A number of polymer damage models have therefore been developed, mostly focusing at the continuum level and using thermodynamical arguments. Domain-transition theories have been applied to describe the softening and Mullins effect of elastomers, ${ }^{16,34,36}$ where the chains are assumed to transit from a hard phase to a soft phase when experiencing large strain. A network alteration theory was proposed Marckmann et al., ${ }^{28}$ where the density of chains is considered to decrease as they transit to the softer domain. This approach was later extended by Zhao ${ }^{57}$ to interpenetrating networks to describe their stiffening and necking behaviors. Diani and 
Merkel $^{9,29}$ further employed the idea of network alternation to describe the Mullins effect and the anisotropy of the network induced by damage. At a more local level, the rupture of chains was related to their stored elastic energy by Lake and Thomas ${ }^{21}$ and recently Mao et al. ${ }^{27}$ Following similar ideas, Volokh ${ }^{49,50}$ proposed the concept of energy limiter, indicating the maximum energy a polymer chain can store before failure. Several models are proposed to describe the time-dependent damage of polymers. Wineman ${ }^{54,55}$ studied various timedependent response of elastomers when they are deformed with high temperatures, at which chain scission and re-formation can be observed in time. Lavoie et al. ${ }^{22}$ adopted the concept of mechanochemistry and described the damage in elastomers under deformation due to rate-dependent scissions. Mao et al. ${ }^{26}$ considered the damage caused by the rupture of ionic bonds and described the viscoelastic damage of the hydrogel under large deformation. Among these models, several failure mechanisms were investigated, including for instance the progressive rupture of the highly stretched chains in multinetwork elastomers, ${ }^{2,52}$ the concurrent roles of covalent and ionic cross-links ${ }^{40}$ in gels, the mechanisms of chain scission, ${ }^{27,41}$ and void growth and coalescence. ${ }^{23}$ These models have been able to explain well-known macroscopic phenomena including the damage-induced stress-softening phenomena (or Mullins effect) ${ }^{15}$ or the partial self-healing observed in certain polymers due to the reattachment of previously inactive bonds. ${ }^{3,7}$

A key to understand these materials relies on our ability to connect the molecular phenomenon of chain stretching and rupture to the macroscopic mechanics. In this context, we have recently introduced a statistical framework that we refer to as the transient network theory or TNT in which the physical state of a polymer network is represented by the distribution $\phi(\mathbf{r})$ of the length and direction of chain end-to-end vector $\mathbf{r}{ }^{45,48}$ An evolution equation was then presented for this distribution, providing a mean to understand the changes in chain configuration within the polymer as a result of deformation and chain detachment and re-formation events. We further showed that the Cauchy stress and energy dissipation could be derived from the second moment of the distribution. We propose here to undertake a similar approach to explore the physics of chain stretch and rupture during polymer failure. As polymer damage often takes place as they undergo large deformation, the model is based on two simple ideas: (a) The force-stretch relation of a single chain strain stiffens at large strains, and this effect becomes more pronounced for smaller chain length.

(b) Chain rupture becomes more and more likely as they reach a critical length that is close to their contour length. Combining these two ideas with the theoretical framework provided by the TNT allows us to explore the damage evolution and resulting stress/strain curves for polymers containing various chain lengths and network characteristics. We particularly explore the case of a multinetwork elastomer and demonstrate that our model is capable of quantitatively predicting the stress/strain relations and hysteresis under large strains, based on which the mechanisms underlying the enhanced energy dissipation are discussed. The effects of key molecular parameters on the macroscopic mechanical behavior are also investigated and discussed.

\section{A STATISTICAL VIEW OF NETWORK DAMAGE}

The collective (or macroscopic) response of a polymer network depends on the individual response and interactions of a large number of constituent chains. For this reason, we start 
our description by considering a statistically representative volume element (or RVE) whose average properties give a good indication of the entire material. Assuming that the chain forms a percolating network, we then give special attention to chains whose points of attachment (or cross-links) are both linked to the network and are as a consequence mechanically active. For the sake of clarity, we further assume that these active chains have a relatively uniform length, characterized by the number $N$ of Kuhn segments. This assumption can later be relaxed when multiple networks are considered. To further attempt to quantify the elasticity of this network, one can count the elastic energy stored in each of the active chain. We will see that this energy depends on the chains end-to-end vector $\mathbf{r}$, which indicates the distance and direction between its attachment point (Figure 1a). In other words, an accurate knowledge of the networks mechanical state at any time $t$ can be gained by knowing the distribution $\phi(\boldsymbol{r}, t)$ of this end-to-end vector for the entire chain population within the RVE. An example of such a distribution when the network is at rest is shown in Figure 1b. This distribution gives two important pieces of information regarding the chain population: first, the integral $c(t)=\int \phi(\mathbf{r}, t) \mathrm{d} \Omega_{\mathrm{r}}$ (where the integral is taken over all chain configurations) is found to be the density of active chain in the RVE. Second, when normalized by the density $c$, the distribution is interpreted as the probability density function $f(\mathbf{r}, t)$ that expresses the likelihood of finding a particular chain at a given configuration $\mathbf{r}$. As a result, the distribution may be rewritten as the product:

$$
\phi(\mathbf{r}, t)=c(t) f(\mathbf{r}, t)
$$

Note that we explicitly included the argument $t$ to emphasize that in the context of damage both the concentration and the probability function depend on time.

\subsection{Distribution Mapping and Damage.}

In the subsequent damage theory, an important concept is the distribution mapping between the chain population in its undeformed configuration and current deformation. To define this mapping, let us consider a single polymer chain whose end-to-end vector is defined by the vector $\mathbf{R}$ when the network is in its undeformed or stress-free state. Let us now imagine that the RVE is subjected to a macroscopic deformation characterized by the deformation gradient tensor $\mathbf{F}$. Considering that the network follows affine deformation, the end-to-end vector of the chain becomes $\mathbf{r}(t)=\mathbf{F}(t) \mathbf{R}$. As a consequence, the end-to-end vector distribution can be expressed in two different ways. On one hand, the current (or stretched) distribution $\phi(\mathbf{r}, t)=\phi(\mathbf{F R}, t)$ portrays the active chain population in its deformed state. On the other hand, the reference distribution $\Phi(\mathbf{R}, t)$ corresponds to the chain population in the absence of deformation (i.e., $\mathbf{F}=\mathbf{I}$ where $\mathbf{I}$ is the identity tensor). This distribution is therefore mathematically obtained by stretching the distribution $\Phi$ according to the tensor $\mathbf{F}$ ${ }^{-1}$ such that $\Phi(\mathbf{R}, t)=\phi\left(\mathbf{F}^{-1} \mathbf{r}, t\right)$. In the remainder of the paper, we will use the reference configuration for its theoretical convenience, but relations with the current configuration will be discussed when appropriate.

When damage is induced to a network, the chain distribution $\Phi$ is affected by the loss of active chains, which may be found in various configurations. To characterize this process, it 
is first useful to define the chain distribution in its undamaged state, immediately after the network has been formed through polymerization. According to statistical mechanics, such a distribution can be expressed in terms of a Maxwell distribution $f_{0}$ with a zero mean and a standard deviation $\sqrt{N} b / 3$ in each of the three spatial directions in the form ${ }^{44}$

$$
\boldsymbol{\Phi}_{0}(\mathbf{R})=c_{0}\left(\frac{3}{2 \pi N b^{2}}\right)^{3 / 2} \exp \left(-\frac{3 \mathbf{R} \cdot \mathbf{R}}{2 N b^{2}}\right)
$$

We note that the classical Maxwell distribution was multiplied by the initial concentration $c_{0}$ to reflect the chain density of the network (i.e., number of chains per unit volume). After loading, the damage induced to the network is represented by a dimensionless damage distribution $\Delta(\mathbf{R}, t)$ describing the fraction of ruptured chains, with undeformed end-to-end vector $\mathbf{R}$, in time. In other words, this function satisfies $\Delta(\mathbf{R}, t) \in[0,1]$, and the reference chain distribution, at any time, can be expressed in terms of the initial distribution $\Phi_{0}(\mathbf{R})$ as

$$
\boldsymbol{\Phi}(\mathbf{R}, t)=\boldsymbol{\Phi}_{0}(\mathbf{R})(1-\Delta(\mathbf{R}, t))
$$

This can also be written in the current state as $\phi(\mathbf{r}, t)=\phi_{0}(\mathbf{r}, t)(1-\delta(\mathbf{r}, t))$ where the stretched damage and chain distributions are defined as $\delta(\mathbf{r}, t)=\Delta\left(\mathbf{F}^{-1} \mathbf{r}, t\right)$ and $\phi_{0}(\mathbf{r}, t)=\Phi_{0}\left(\mathbf{F}^{-1} \mathbf{r}\right)$. From this definition, it is further possible to define a global damage measure $D$ by integrating the damage distribution over all chain configurations:

$$
D=\frac{1}{c_{0}} \int \boldsymbol{\Phi}_{0} \Delta \mathrm{d} \Omega_{R}=1-\frac{c}{c_{0}}
$$

where the last term can easily be obtained from (3). It is clear that this scalar ranges from 0 in the absence of chain damage (i.e., $c=c_{0}$ ) to unity when the network is fully damaged (i.e., $c=0$ ). For clarity, the mapping of the damaged chain distribution is illustrated in Figure 2 for a simple one-dimensional case. From a physical point of view, we note that chain rupture can occur in the middle of a chain (chain scission) or at their extremities (cross-link failure), depending on the strength of the bonds. In this paper, we do not specify which mechanism is predominant, but we postulate that from a mechanical point of view their consequence is a similar reduction in the concentration of connected chains, dissipation of the elastic energy, and softening of the network.

\subsection{Stored Elastic Energy and Stress.}

Based on the above statistical description, let us now assess the elastic energy stored in the network. In this context, Kuhn and Grun ${ }^{20}$ estimated the entropy of a chain by idealizing them as a series of jointed segments undergoing a random walk (freely jointed chain model). If we recall that the mean end-to-end distance of a chain at zero force is $N b$ where $N$ and $b$ are the number and length of Kuhn segments in a chain, respectively, the stretch ratio can be 
defined as $\lambda=r /(\sqrt{N} b)$ where $r=|r|$ is the end-to-end distance. The cost of chain stretching can be expressed in terms of the potential: ${ }^{44}$

$$
\psi(r)=k_{\mathrm{B}} T N\left(\frac{\lambda}{\sqrt{N}} \beta+\frac{\beta}{\sinh \beta}\right) \quad \text { where } \beta=\mathscr{L}^{-1}\left(\frac{\lambda}{\sqrt{N}}\right)
$$

where $k_{\mathrm{B}}$ is Boltzmann's constant, $T$ is the absolute temperature, and $\mathscr{L}^{-1}$ is the inverse Langevin function, such that $\mathscr{L}(\beta)=\operatorname{coth}(\beta)-1 / \beta$. If the chain distribution is known, it is possible to estimate the elastic energy density stored in the network by integrating $\psi(\mathbf{r})$ over all configurations. Doing so, and introducing the difference $\Delta \psi$ between the network in its current and stress-free state, we obtain

$$
\Delta \Psi=\int[\phi-\boldsymbol{\Phi}] \psi \mathrm{d} \Omega_{r}+p \frac{\Delta \nu}{\nu}
$$

where $v$ is the specific volume of the polymer network and the integral is taken over the chain's configuration space $\Omega_{r}=\left\{r \mid r \in \mathbb{R}^{3}\right\}$. Note that the Lagrange multiplier $p$ enforces the incompressibility conditions that the specific volume $v$ of the network remains equal to its initial value $v_{0}$ at all times (i.e., $\Delta v=V-v_{0}=0$ ). Using energy conservation, we can further show (see next section) that the Cauchy stress $\sigma$ in the polymer network can be found by integration as follows:

$$
\boldsymbol{\sigma}=\int[\phi-\boldsymbol{\Phi}](t \otimes \mathbf{r}) \mathrm{d} \Omega_{r}+p \mathbf{I}
$$

where $\mathbf{t}=\mathrm{d} \psi / \mathrm{d} \mathbf{r}$ is the chain force vector in a single chain. This expression provides a bridge between the molecular scale (force, stretch, and orientation of single chains) and its macroscopic response. Finally, using the separation of the distribution function (see eq 1) into the active chain concentration and normalized probability functions $F$ (reference state) and $f$ (stretched state), the stress can equivalently be written as $\boldsymbol{\sigma}=c \int_{r}[f-F](t \otimes \mathbf{r}) \mathrm{d} \Omega_{\mathrm{r}}+p \mathbf{I}$ where $f$ and $F$ are the probability density function in the current and reference state, respectively. Interestingly, if one rewrites the current chain concentration as $c=c_{0}(1-\mathrm{D})$, the stress can be written as

$$
\boldsymbol{\sigma}=(1-D) \boldsymbol{\sigma}_{0}+p \mathbf{I}
$$

where $\sigma_{0}=c_{0} \int[f-F](\mathbf{t} \otimes \mathbf{r}) \mathrm{d} \Omega_{r}$ is the damage-free stress. In other words, the damage parameter induces a drop in stress from its original undamaged level. This expression is reminiscent of empirically damage mechanics model described in refs 22 and 57. Finally, we note that eq 7 can be calculated numerically. For a 3D problem, we first discretize the chain space $\Omega_{r}$ with a uniform grid of $(n \times n \times n)$ points. The integration is then approximated on 
the grid by a trapezoidal or Simpson's integration scheme based on the value of integrands on the grid points. Readers are referred to Chapra ${ }^{4}$ for detailed introduction on different types of integration schemes.

\section{DAMAGE EVOLUTION IN POLYMER NETWORKS}

An advantage of the statistical approach in modeling damage is that it is directly formulated in terms of a failure criterion at the chain level. Indeed, the quantity $\Delta(\mathbf{R}, t)$ appearing in (3) is interpreted as the probability of failure of a chain that is originally characterized by an end-to-end vector $\mathbf{R}$. Since one usually knows the macroscopic deformation history through the deformation gradient $\mathbf{F}(t)$, it is possible to track the chain's stretch history $\mathbf{r}=\mathbf{F}(t) \mathbf{R}$ and thus its chances of failure via a molecular-motivated criterion. In this section, we therefore construct a simple and thermodynamically consistent model for chain failure that directly affect the damage response of the polymer through its stress response.

\subsection{Form and Conditions on the Damage Evolution Law.}

The phenomenon of material damage is a history-dependent process, which mostly depends on deformation path of a volume element over time. For this reason, our formulation should follow an incremental form, which involves the rate $\dot{\Delta}$ of the chain damage probability. For each continuum point, the state of the network is described by the distribution function $\phi(\mathbf{r}, t)$ that is defined in the chain space spanned by the end-to-end vector $r$. Its evolution results from the interplay between two mechanisms: (i) the loss of chains due to rupture and (ii) the stretch of chains due to network deformation. With this consideration, the evolution of chain distribution is associated with the rate of damage $\dot{\delta}$ (or $\dot{\Delta}$ in the reference configuration) by eq 3 as

$$
\frac{D \phi(\mathbf{r}, t)}{D t}=-\mathbf{\Phi}_{0} \dot{\delta}
$$

where the material time derivative $\frac{D \phi(\mathbf{r}, t)}{D t}=\frac{\partial \phi}{\partial t}+\dot{\mathbf{r}} \cdot \nabla_{r} \phi$, with $\nabla_{r} \phi=\partial \phi / \partial \mathbf{r}$, accounts for the distortion of $\phi$ in time due to the stretch of chains (see the Appendix A.1 for a more detailed explanation). Assuming affine deformation, the rate of chain's stretch can be associated with macroscopic deformation as $\dot{\mathbf{r}}=\mathbf{L r}$, where $\mathbf{L}=\dot{\mathbf{F F}} \mathbf{}^{-1}$ is the velocity gradient. 45 After further calculations, the material time derivative can be rewritten as

$$
\frac{D \phi}{D t}=\frac{\partial \phi}{\partial t}+\mathbf{L}:\left(\nabla_{r} \phi \otimes \mathbf{r}\right)
$$

We make two observations regarding eq 10. First, for a purely elastic network, i.e., there is no chain rupture, the change in chain distribution only arises from distortion, and the righthand side of eq 9 vanishes. Second, this equation is consistent with earlier version of the transient network theory (TNT) used to describe polymer viscoelasticity. ${ }^{45,48}$ In this context, we identify the term $\phi_{0} \dot{\delta}$ is the rate of chain failure, while no chain reattachment events 
occur in a pure damage formulation (i.e., without self-healing). We finally note that the evolution of the distribution $\Phi(\mathbf{R}, t)$ at the reference configuration follows eqs 9 and 10 in the particular case when $\mathbf{F}=\mathbf{I}$ (or equivalently $\mathbf{r}=\mathbf{R}$ ).

To be thermodynamically consistent, let us now seek an expression of the second principle for an isothermal process. For this, we evaluate the change in the elastic energy $\Delta \psi$ as the polymer network undergoes an incremental macroscopic deformation represented by the velocity gradient $\mathbf{L}$. The general case, including changes in temperature, was described in a previous study ${ }^{45}$ and is therefore not shown for brevity. In this case (isothermal process), the second principle states that the energy dissipation $\mathscr{D}$ is equal to the difference between the work done by internal forces and the change in deformation energy as

$$
\mathscr{D}=\boldsymbol{\sigma}: \mathbf{L}-\Delta \dot{\boldsymbol{\Psi}} \geq 0
$$

where $\boldsymbol{\sigma}$ is the Cauchy stress. As seen in eq 11 , the second principle is enforced by the fact that $\mathscr{D}$ must remain positive at all times. To proceed further, we use (6) to estimate the rate of change in elastic energy $\Delta \dot{\Psi}$ (details shown in Appendix A.2) and obtain

$$
\Delta \dot{\boldsymbol{\Psi}}=\left[\int(\phi-\mathbf{\Phi})(\mathbf{t} \otimes \mathbf{r}) \mathrm{d} \Omega_{r}+p \mathbf{I}\right]: \mathbf{L}-\int\left(\phi_{0} \dot{\delta}-\mathbf{\Phi}_{0} \dot{\Delta}\right) \psi \mathrm{d} \Omega_{r}
$$

Now using the definition of stress in (7) and identifying the terms with (11) leads us to the energy dissipation:

$$
\mathscr{D}=\int\left(\phi_{0} \dot{\delta}-\mathbf{\Phi}_{0} \dot{\Delta}\right) \psi \mathrm{d} \Omega_{r} \rightarrow \dot{\Delta}(\mathbf{R}, t) \geq 0
$$

Using the fact that the strain energy function $\Psi$ remains positive for all values of $\mathbf{r}$, the positiveness of the dissipation is true in the condition that the local detachment rate remains positive at all time (as expressed in the right-hand side of eq 13). From a physical viewpoint, eq 13 expresses the idea that energy dissipation in the network arises from the loss of elastic energy resulting from the rupture of stretched polymer chains.

\subsection{Chain Failure Evolution Law.}

To derive a damage evolution law, we turn to a probabilistic description of chain failure. Typically, the rupture of a chain occurs when the internal energy due to bond stretching exceeds a critical value. ${ }^{21} \mathrm{~A}$ direct consideration of this mechanism, however, requires a detailed modeling of the bond deformation at the molecular level, which may complicate the proposed approach and introduce expensive computational cost. In this paper, we focus on establishing a connection between chain mechanics and the global response; we therefore consider a simpler phenomenological criterion for chain (or bond) rupture. For this, as noted by Thomas ${ }^{42}$ and later by Mao, ${ }^{27}$ there exists a characteristic length that is associated with the critical internal energy for rupture. For elastomers, this characteristic length is postulated 
as $l \simeq \sqrt{N} b \cdot{ }^{27}$ On the basis of this consideration, we assume that the rupture of a single chain occurs when it exceeds a limited end-to-end distance $r_{\mathrm{c}}$ above which failure occurs. When considering a large population of chains, a variability exists in this limit stretch due to small differences in chain orientation, length, and configurations within the network. In this study, this variability is expressed in terms of a cumulative probability function $P(r)$ that expressed the likelihood of failure of a chain that experiences an end-to-end distance $r$. Assuming Gaussian statistics, we write

$$
P(r)=\frac{1}{\sqrt{2 \pi} \sigma_{\mathrm{d}}} \int_{0}^{r} \exp \left[-\frac{1}{2 \sigma_{\mathrm{d}}^{2}}\left(\xi-r_{\mathrm{c}}\right)^{2}\right] \mathrm{d} \xi
$$

where the standard deviation $\sigma_{\mathrm{d}}$ expresses the variability in chain failure. As shown by Figure 3, the failure probability is extremely low at low stretch while it dramatically increases as the end-to-end distance $r$ approaches $r_{\mathrm{c}}$. As expected, the failure probability asymptotes to $P=1$ for large stretches (above $r_{\mathrm{c}}$ ). We finally note that the function (14) can be rewritten in terms of the reference end-to-end distance $R$ via the mapping $\mathbf{r}=\mathbf{F R}$.

A damage evolution law can then be constructed from the fact that chains begin to rupture as they are stretched beyond their failure point. To understand how this statement is translated into a mathematical formulation, consider a population of chains with an original end-to-end vector $\mathbf{R}$, original concentration $\Phi_{0}(\mathbf{R})$, and damage fraction $\Delta(\mathbf{R}, t)$. Let us now assume that the network is stretched according to a macroscopic deformation gradient $\mathrm{F}$, such that the chains are all stretched to an end-to-end vector $\mathbf{r}=\mathbf{F R}$. To estimate whether those chains will experience additional damage, we consider the following cases:

- The probability of chain failure $P(\mathbf{r})$ is smaller than or equal to the current damage $\delta(\mathbf{r})$ in this population. In this case, all weaker chains have already ruptured and no additional damage occurs. In other words, if $P(\mathbf{F R}) \leq \Delta(\mathbf{R}, t)$, the damage evolution equation becomes

$$
\dot{\Delta}(\mathbf{R}, t)=0
$$

- The probability of chain failure $P(\mathbf{r})$ is larger than the current damage $\delta(\mathbf{r}, t)$ in this population. In this case, a portion of the chains break such that the current damage $\delta(\mathrm{r}, t)$ becomes equal to the failure probability $P(\mathbf{r})$. In this case, the increase in damage can be determined as

$$
\dot{\Delta}(\mathbf{R}, t)=\left.\dot{P}\right|_{\mathbf{R}}=\nabla P(\mathbf{r}) \cdot \dot{\mathbf{r}} \geq 0
$$

with $\dot{\mathbf{r}}=\dot{\mathbf{F} R}$ and $\left.\dot{P}\right|_{\mathbf{R}}$ is used to represent the change in failure probability for a constant reference end-to-end vector $\mathbf{R}$. From eq 13, damage can only evolve if the right-hand side is positive. In other words, if $P(\mathbf{F R})>\Delta(\mathbf{R}, t)$, the damage evolution equation becomes 
$\dot{\Delta}(\mathbf{R}, t)=\nabla P(\mathbf{r}) \cdot \dot{\mathbf{r}} \quad$ if $\nabla P(\mathbf{r}) \cdot \dot{\mathbf{r}} \geq 0$

$$
\dot{\Delta}(\mathbf{R}, t)=0 \quad \text { if } \nabla P(\mathbf{r}) \cdot \dot{\mathbf{r}}<0
$$

The first condition corresponds to the case of loading; i.e., most chains stretch above their critical value and induce damage in the network. The second condition corresponds to the case of unloading; i.e., the chain stretch decreases and therefore cannot accumulate additional damage.

Initial Conditions.-In its initial state (i.e., $t=0$ ), the polymer may often be considered stress-free. This means that the deformation gradient is $\mathbf{F}(0)=\mathbf{I}$ where I is the identity tensor. In this condition, we therefore have $\mathbf{r}=\mathbf{R}$, and the condition for no damage evolution in the stress-free configuration is according to (15):

$$
\Delta(\mathbf{R}, 0)=P(\mathbf{r})
$$

This completes the statistical description of chain damage in the network. In addition, the chain distribution at the reference configuration follows a Gaussian distribution, indicating that a small number of chains possess very large end-to-end distance. An initial damage is applied on these chains since their end-to-end distances is much greater than the critical value for rupture $\left(r \gg r_{c}\right.$ ). With this consideration, the chain distribution in the initial state reads

$$
\phi(\mathbf{r}, 0)=\phi_{0}(\mathbf{r})(1-P(\mathbf{r}))
$$

\subsection{A Simple Illustration.}

To help illustrate the statistical damage model, we consider the cyclic uniaxial tension of a cylindrical sample that is composed by a single elastic network, as shown in Figure 5. This sample is subjected to two consecutive cycles (as shown in Figure 5a) with maximum elongation $\lambda=2.5$ and 3.4. In Figure 5b, we show the isocontours of the chain distribution $\phi$ in the $x-y$ section at different time point during deformation. As expected, this surface initially takes a spherical shape, indicating that the polymer network is isotropic. After the sample is stretched along the $x$-axis, the distribution becomes elliptic as the chains stretch along the $x$-direction and become more compressed along the $y$-direction as a result of incompressibility. As the distribution intersects the damage region (represented by dashed circle), damage is induced to the highly stretched chains. As a result, the distribution becomes truncated along the $x$-direction, and becomes nonspherical as the deformation is removed. The same scenario occurs during the second loading, but we note that damage is only initiated once the deformation reaches the same level as that in the end of the first cycle (point $\mathrm{D}$ ). Beyond this point, however, the distribution is further stretched and damaged, 
resulting in a more highly truncated sphere after the deformation is removed (point F). To better understand the damage mechanism, a one-dimensional ( $x$-projection) representation of the distribution and its evolution are finally given in Figure 5c. For comparison, we show here its representation in the original $\left(\phi_{0}(\mathbf{r})\right)$ and damaged state $(\phi(\mathbf{r}, t))$, together with the failure probability function $P(\mathbf{r})$.

To further appreciate how damage accumulates during deformation, Figure $5 \mathrm{~d}$ depicts the variable $D$, computed by eq 4 , as a function of time. We see that in the first cycle damage accumulates during the loading stage and stalls during the unloading. In the second cycle, during the time $C-D$, the deformation of the sample is smaller than the maximal deformation in the first cycle; therefore, all brittle chains are already ruptured during the first cycle, and no additional damage is observed until point $D$, where the sample regains the maximum stretch of the previous cycle. For clarity, Figure 5 a also shows the evolution of the damage $\Delta(R, t)$ (insets) with green dashed lines and compares it to the initial function $\Delta(R, 0)$ depicted by a black dotted line. Initially, at point $A$, these two curves overlap, consistently with eq 19. As damage accumulates, however, $\Delta(R, t)$ evolves toward $R=0$. This narrowing of $\Delta(R, t)$ may be interpreted by the fact that as the polymer network is stretched over its load-carrying capacity, chains with an initially large end-to-end distance reach their critical stretch $\lambda_{\mathrm{c}}$ and rupture first. In contrast, chains that are originally shorter survive damage, i.e., $\Delta(R, t) \approx 0$.

Finally, we determine the stress and overall damage in the specimen using eqs 7 and 4, respectively. For many experimental measurements, the results are shown in terms of nominal stress $\boldsymbol{\sigma}_{N}=\boldsymbol{J}^{-1} \boldsymbol{\sigma}$ where $J=\operatorname{det}(\mathbf{F})=1$ for an incompressible polymer. Figure $5 \mathrm{~d}$ thus depicts the predicted normal nominal stress along the $x$-direction as a function of stretch $\lambda$. We observe that in the first cycle the stress-strain curve takes different paths between the loading and the unloading stage. We clearly observe a decrease in the network's stiffness due to the loss of active chains resulting from damage depicted in Figure 5d. During the second cycle, the loading path first closely follows the unloading of the cycle 1 and appears to continue the loading curve of cycle 1 after point $D$, where new damage starts to accumulate (Figure 5e). This behavior is reminiscent of the Mullins effect observed in a number of filled rubbers ${ }^{10}$ and multiple-network polymers. ${ }^{52}$ We further see that as damage increases, the polymer's stiffness decreases until the curve reaches a maximum. This point marks the onset of strain localization that usually takes the form of the necking of a cylindrical specimen in tension.

We finally note that the mechanical response of the material depends on our choice of $r_{\mathrm{c}}$ and $\sigma_{\mathrm{d}}$. To identify their roles, we performed a parameter study and whose outcomes are shown in Appendix A.3. We find that an increase in $r_{\mathrm{c}}$ leads to a rise in toughness and stiffness of as well as less cumulative damage. This is because chains with larger $r_{\mathrm{c}}$ rupture in a higher stretched state and dissipate more energy during deformation. Regarding the role of $\sigma_{\mathrm{d}}$, we find that increasing $\sigma_{\mathrm{d}}$ also improves both the stiffness and the toughness of the network. However, its effect on the cumulative damage is insignificant. 


\section{APPLICATION TO THE DAMAGE MECHANICS OFMULTINETWORK ELASTOMERS}

Multinetwork elastomers present interesting systems that can be adequately designed to significantly increase the fracture resistance of soft materials. ${ }^{13,52}$ Generally, these polymers consist of a brittle network made of small and stiff chains, reinforced by other "tough networks" made of longer and softer chains. As a result, when the polymer is deformed, the brittle network breaks first, creating multiple cracks that are bridged by the tough network, leading to a significant improvement in toughness. ${ }^{11,31,52}$ To achieve a controllable reinforcement protocol, efforts have been devoted to quantifying the effect of the chain rupture and network charateristics on polymer toughening. ${ }^{11,30}$ Here we demonstrate that the presented theory provides a useful tool to connect the macroscopic behavior of these materials to their molecular design, with a potential to assist and guide experimental efforts.

Synthesis of the multinetwork elastomer is achieved by a sequential polymerization technique, as shown in Figure 6a. In the experiment, ${ }^{11}$ a single network (SN) elastomer is first synthesized via UV polymerization using the monomer ethyl acrylate (EA). To introduce an additional network, the elastomer is then preswelled in the EA monomer solution which effectively stretches the existing chains in an isotropic manner. By adding a certain amount of cross-linkers and UV initiators, an additional loosely cross-linked network is then formed. Following this strategy, double-network (DN) and triple-network (TN) elastomers can be fabricated. In Figure 6b, we show the chain distribution $\phi$ corresponding to each network (referred to by the superscripts 1,2, and 3) after three consecutive steps of polymerization. We see here that when an additional network is added, the chains in the existing networks are subjected to an increase in the degrees of prestretching. As a result, their chain distributions become wider and closer to the failure probability function $P(r)$, as shown by the evolution of $\phi^{1}$ from the SN to TN elastomer.

Two examples are presented. We first calibrate the model parameters by comparing with the experimental work of Ducrot et al. ${ }^{11}$ and explain the physical mechanism of the mechanical reinforcement by analyzing the behavior of each individual network. Second, we study the physical mechanism behind the necking instability of these elastomers when they are highly stretched $(\lambda>5)$. We also discuss how the necking phenomena can be controlled by tuning the network properties during the polymerization.

\subsection{Mechanical Reinforcement of Multiple Network Elastomers.}

Cyclic uniaxial tensile loadings were then performed on both DN and TN elastomers. To obtain a fairly close fit to experimental measurements for these two elastomers, we identify that the number of Kuhn segments in the chains of each network are $N_{1}=45$ and $N_{2}=N_{3}=$ 200. Besides, since the monomer used in each network is the same, the damage criteria of the chains are identified as $r_{\mathrm{c}}=0.87 \mathrm{Nb}$ and $\sigma_{\mathrm{d}}=0.01 r_{\mathrm{c}}$. In Figure $7 \mathrm{a}$, we show the true tensile stress $\sigma$ as a function of stretch $\lambda$ (experimental data converted from ref 11) for the $\mathrm{DN}$ elastomer. We see that the stress-stretch relationship of the elastomer is linear at small stretch $(\lambda<2)$ and stiffens upon further elongation. Besides, a very small hysteresis is observed during the loading cycles, indicating that the energy dissipated by chain rupture in 
the DN elastomer is insignificant. This is also revealed by the evolution of the chain distribution shown by the snapshots in the subfigures. We see that the majority of chains remain within the boundary delimited by the chain failure function $P(\mathrm{r})$ during deformation. As a result, most chains survive from being damaged, and no visible truncation is observed in the chain distribution of both networks. This is also observed in the evolution of the damage parameter $D$ for each network (Figure $7 \mathrm{~b}$ ) as a function of $\lambda$, where the damage accumulated in the first network is only $D \approx 0.8 \%$ at the maximum stretch.

The TN elastomer shows a different behavior during the cyclic tensile test, as shown in Figure 8a. First, the elastomer stiffens at a much smaller stretch $(\lambda \approx 1.5)$ and softens when $\lambda>2.5$. Second, during the loading history, the hysteresis starts to become visible at $\lambda \approx 1.5$ and becomes more significant as the deformation increases. In Figure 8b, we further decompose the stress $\sigma$ into contributions from each network, denoted by $\sigma^{1}, \sigma^{2}$, and $\sigma^{3}$. Although the weight fraction of the first network is small $(6 \%)$, it is the main contributor to the macroscopic response while the stress in the second and the third network is almost negligible. To further reveal the molecular physics behind this phenomenon, we track the evolution of the chain distribution of each network in Figure 8c. Because of the higher level of prestretching, the chains in the first network are more tightly connected with a distribution $\phi^{1}$ that is wide compared to that in the DN at the initial time (point $\mathrm{A}$ ). Therefore, the chains approach their rupture length $r_{\mathrm{c}}$ at a smaller stretch ratio, as shown by the snapshots $A-C$, and stiffen. After being further stretched, these chains break, as shown by the snapshots $C$ $E$, resulting in the softening of the polymer. Besides, $\phi^{2}$ and $\phi^{3}$ remain narrow during the history of deformation, indicating that the chains in these two networks remain loosely connected and therefore have insignificant contribution to the mechanical response and chain damage (see Figure 8b,d). As a summary, in comparison with the DN elastomer, the TN elastomer exhibits an increased initial stiffness at small strain and hysteresis at large deformations. We note that in experiment identifying the roles of each network is not trivial and usually requires the construction of a master curve from the stress-strain curves of different samples. ${ }^{30}$ However, by use of the TNT theory, the individual response of each network can be straightforwardly obtained, where one clearly sees that the prestretching of the first network plays the key role in this mechanical reinforcement.

\subsection{Necking Phenomena of Multiple Network Hydrogels.}

When subjected to a higher degree of stretching (e.g., $\lambda>5$ ), the multinetwork hydrogels ${ }^{32,33,51}$ have been found to exhibit necking. Similar necking behavior has also been observed in a multinework elastomer system recently. ${ }^{30}$ Necking occurs when the tensile deformation localizes in an initially narrow zone, which may widen upon further deformation. When this phenomenon occurs, the nominal stress remains almost constant as the necked region grows until it occupies the entire sample. Once the neck has taken over the entire sample, the polymer deformation returns to a homogeneous state, and the stress can increase again. Importantly, after necking, the elastomer becomes very stretchable and is able to sustain an elastic elongation of about several times its original length. Multinetwork elastomers have been shown to exhibit superior ductility during necking, making them more fit for applications that required high extensibility. 5,35 To better understand the physics behind this phenomena, we employ the proposed statistical framwork and explore the 
necking of a quadruple-network (QN) elastomer, which was previously studied experimentally by Millereau. ${ }^{30}$ This QN elastomer is synthesized using the same monomer, EA, as the DN and TN discussed in section 4.1 and the same sequential polymerization technique introduced in Figure 6a. After being fabricated, the first network is tightly crosslinked and highly stretched. According to ref 30 , the weight fraction and the prestretch ratio of each network are $c_{\mathrm{wt} \%}^{1}=2.88 \%, c_{\mathrm{wt} \%}^{2}=10.94 \%, c_{\mathrm{wt} \%}^{3}=33.40 \%, c_{\mathrm{wt} \%}^{4}=52.47 \%$ and $\lambda_{\text {pre }}^{1}=3.42, \lambda_{\text {pre }}^{2}=2.04, \lambda_{\text {pre }}^{3}=1.34, \lambda_{\text {pre }}^{4}=1$.

Figure 9a shows the experimental measurement and model prediction of the stress-stretch $\left(\sigma_{N^{-}} \lambda\right)$ relationship of the QN hydrogel where we used the same molecular parameters as those for the DN and TN elastomers without any additional fitting parameters. To better interpret these results, we divide the stress-strain curve into three regions: (i) the first stiffening region $\left(\lambda<\lambda_{U}\right)$, (ii) the necking region $\left(\lambda_{U}<\lambda<\lambda_{N}\right)$, and (iii) the second stiffening region $\left(\lambda>\lambda_{N}\right)$. In the first stiffening region, the elastomer deforms homogeneously, and the chains in the first network are stretched near their maximum end-toend distance, leading to macroscopic stiffening. Upon additional deformation, the rupture of these chains triggers a decrease in the polymer's stiffness and subsequent softening after the peak stress is reached. In the necking regime, assuming that the sample deformation remains homogeneous, the model predicts that the $\sigma_{N^{-}} \lambda$ relationship is no longer monotonic, where $\sigma_{N}$ first decreases due to the rupture of the first network and increases again when the chains in the second network stiffen. However, due to the nonmonotonic change in $\sigma_{N}$ with respect to $\lambda$, the sample becomes unstable and exhibits strain localization. ${ }^{46,51}$ Thus, instead of deforming uniformly, the sample spontaneously separates into two phases: the necked phase with stretch ratio $\lambda_{N}$ and the un-necked phase with stretch ratio $\lambda_{U}$. In this regime, the nominal stress $\sigma_{N}^{N}$ can be determined by equating the two shaded areas shown in Figure 9a,b according to Maxwell's rule of phase transition. ${ }^{17}$ As a result of this instability, in experiment, one would observe that the sample first elongates homogeneously with monotonous increase in stress until we reach the onset of necking at $\lambda=\lambda_{N}$. Two coexisting stretch ratios $\left(\lambda_{U}\right.$ and $\left.\lambda_{N}\right)$ are then seen along the sample until the second stiffening starts.

Overall, the presented model quantitatively captures the experimental data, specifically the stable loading branch, the necking stress, and the unloading branch. However, we also observe two discrepancies that are worth further discussion. First, the experimental data show that the necking starts before the peak stress predicted by our model is reached. This can be attributed to pre-existing defects (e.g., voids) in the sample around which the concentrated stress may trigger necking. Second, our model overestimates $\lambda_{N}$ and underestimates the stress for the second stiffening branch. A possible reason is that we used the same chain lengths as the DN and TN hydrogels. However, the effective chain length for the second network in the QN hydrogel may be smaller than $N_{2}=200$ due to interactions between the second network and the third or fourth networks. This motivates us to remove the constraint $N_{2}=200$ and treat it as a fitting parameter. Excellent agreement with experimental data can thus be found if $N_{2}=170$ (see Figure 9b). To further investigate the role of each network in the polymer behavior, Figure $9 \mathrm{c}$ shows the $\sigma_{N^{-}} \lambda$ curve for each network independently. This results clearly shows that the first network is responsible for the 
first stiffening and the subsequent softening of the sample, while the second network plays the main role in the second stiffening. We also see that chain damage accumulates as the necked zone propagates, as revealed by the hysteresis in the necking regime. To quantify the chain loss, Figure $9 \mathrm{~d}$ then plots the chain damage $D$ of each network as a function of stretch ratio $\lambda$. We see that the damage during the deformation is mainly due to the rupture of the first network while failure in other network is negligible (as shown in the subfigure of Figure 9d). The integrity of the longer chains indeed ensures that the hydrogel possesses a significant elastic extensibility after necking.

With this calibrated model, we finally predict how the polymer response varies as a function of the molecular design of each networks. For this, it is first important to note (from Figure $9 \mathrm{~b})$ that the first and second networks are the main two players in the range of stretch under investigation. We therefore restrict our study to a double-network (DN) sample and investigate the role of the first network on its mechanical response. Experimentally, the chain property can be controlled in three aspects: the chain concentration (weight fraction $c_{1}$ ), the prestretch ratio $\lambda_{\text {pre }}$ applied on the first network, and the chain length (segment number $N_{1}$ ). Here, we consider an elastomer of properties $c_{\mathrm{wt} \%}^{1}=0.05, \lambda_{\text {pre }}=1.8$, and $N_{1}=45$ as reference sample and study the effect of varying these three parameters independently, keeping the chain length of the second network constant $\left(N_{2}=170\right)$. Figure 10a shows the effect of chain concentration on the uniaxial stress-strain response of the polymer, in which it is clear that the first stress stiffening becomes more significant as the molar fraction of the first network $c_{\mathrm{wt} \%}^{1}$ increases. As a result, the necking instability becomes more apparent, with a sharper drop in stress after after the peak stress is reached. In addition, although the peak stress increases as $c_{\mathrm{wt} \%}^{1}$ becomes larger, its horizontal position remains unchanged, as indicated by the arrow showing its translation. This means that varying $c_{\mathrm{wt} \%}^{1}$ does not affect the stretch at which the necking instability sets in. In contrast, Figure 10b shows that by varying the prestretch ratio $\lambda_{\text {pre }}$, one can control whether necking occurs. For small values of $\lambda_{\text {pre }}\left(\lambda_{\text {pre }}=1.1\right)$, the first and second stiffening overlap, and no necking is observed. However, as $\lambda_{\text {pre }}$ increases, the necking is observed and the peak shifts to the left, implying that the stiffening of the first network occurs at a smaller $\lambda$. Lastly, Figure 10c shows that shorter chains (small $N_{1}$ ) in the first network can promote the necking instability, since shorter chains stiffen earlier. As $N_{1}$ increases, the peak stress is shifted to the right and necking vanishes when $N_{1}=90$, which is about 0.53 of the chain length of the second network.

\section{CONCLUSION}

As a summary, we proposed a statistical framework to describe the damage of polymeric materials based on their internal molecular structure. We have shown that this approach provides a clear connection between molecular details, such as chain length, density, and force to failure, and the combination of hyperelasticity and damage at the macroscopic scale. More specifically, based on a simple chain failure criterion (chain ruptures at a critical length), the model revealed fairly complex macroscopic behaviors that includes hysteresis, the Mullins effect, stress stiffening/softening, and necking. Despite its simplicity, the 
proposed approach could further reproduce a number of experimental results regarding the failure of multinetwork elastomers and confirm the micromechanisms responsible for energy dissipation and toughnening. In this context, we found that the necking phenomenon occurring during unaxial testing could be controlled via a fine-tuning of the chain properties during polymerization. These theoretical predictions are therefore useful for both improving our fundamental knowledge of polymer failure and for practical applications that aim for durable and fracture resistant polymer. Such tough and functional materials are indeed very desirable for tissue engineering, $, 1,8,39$ soft robotics, ${ }^{37,47}$ soft armors, ${ }^{53}$ and soft elastomers. ${ }^{25}$ We note that the criterion for chain rupture introduced in this paper is phenomenological. To gain more physical insights, this criterion can however be improved with a more detailed modeling at the level of a bond. This can be included, for instance, relaxing the rigidity assumption of the Kuhn segments and evaluating the rupture based on the stored elastic energy in a bond due to stretching. ${ }^{26,27}$ One finally notes that while this paper focuses on the damage of covalently cross-linked polymers, the statistical framework provides an attractive avenue to explore damage in networks formed by physical cross-links ${ }^{45,48}$ (e.g., ionic/ hydrogen bonds). Because of the dynamic nature of these bonds, chains have the ability to dissociate and re-form in time, resulting in the viscoelasticity in the bulk response and that phenomenon of self-healing. Future efforts can therefore include the study of the mechanical behaviors of these types of materials, such as a tough ionic hydrogel, ${ }^{40,56}$ showing a combination of viscoelasticity, damage, ${ }^{18,38}$ and fracture. ${ }^{19}$

\section{ACKNOWLEDGMENTS}

The author acknowledges the support of the National Science Foundation under the NSF CAREER Award 1350090. Research reported in this publication was also partially supported by the National Institute of Arthritis and Musculoskeletal and Skin Diseases of the National Institutes of Health under Award 1R01AR065441. The content is solely the responsibility of the authors and does not necessarily represent the official views of the National Institutes of Health.

\section{APPENDIX}

\section{A.1.: Material Time Derivatives of the Chain Distribution Function}

The material time derivative of the chain distribution $\phi(\mathbf{r}, t)$ can be defined as its rate of change as one follows a particular chain with initial end-to-end vector R. Mathematically, this idea is expressed as

$$
\frac{D \phi(\mathbf{r}, t)}{D t}=\left.\frac{\partial \phi}{\partial t}\right|_{\mathbf{R}=\mathrm{const}}
$$

Using the fact that $\mathbf{r}=\mathbf{r}(\mathbf{R}, t)$, or $\phi=\phi(\mathbf{r}(\mathrm{R}, t), t)$, and invoking the chain rule, we obtain

$$
\frac{D \phi}{D t}=\frac{\partial \phi}{\partial \mathbf{r}} \frac{\partial r(\mathbf{R}, t)}{\partial t}+\frac{\partial \phi}{\partial t}
$$

If we further assume that chain deformation obeys the affine assumption (i.e., $\dot{\mathbf{r}}=\mathbf{L r}$ ), we finally write 


$$
\frac{D \phi}{D t}=\left(\nabla_{r} \phi \otimes r\right): \mathbf{L}+\frac{\partial \phi}{\partial t}
$$

\section{A.2.: Derivation of Variation of Energy}

According to eq $6, \Delta \dot{\Psi}(\mathbf{r})$ can be computed as

$$
\Delta \dot{\boldsymbol{\Psi}}=\int(\dot{\phi}-\dot{\boldsymbol{\Phi}}) \psi \mathrm{d} \Omega_{r}+p \operatorname{tr}(\mathbf{L})
$$

We first evaluate the term corresponding to the current configuration:

$$
\dot{\Psi}=\int \dot{\phi} \psi \mathrm{d} \Omega_{r}
$$

According to eqs 9 and 10, the change in chain distribution takes the form $\dot{\phi}=-\mathrm{L}:(\nabla \phi \otimes \mathbf{r})-\phi_{0} \dot{\delta}$. Using this relationship, one can write eq 22 as

$$
\dot{\boldsymbol{\Psi}}=\int-\mathbf{L}:(\nabla \phi \otimes \mathbf{r}) \psi \mathrm{d} \Omega_{r}-\int \phi_{0} \dot{\delta} \mathrm{d} \Omega_{r}
$$

To proceed further, we use the integration by part and the divergence theorem for the first integral and obtain

$$
\begin{gathered}
\int-\mathbf{L}:(\nabla \phi \otimes \mathbf{r}) \psi \mathrm{d} \Omega_{r}=-\mathbf{L}: \int \mathbf{n} \cdot(\mathbf{r} \phi \psi) \mathrm{d} S_{r} \\
+\int \operatorname{tr}(\mathbf{L}) \phi \psi \mathrm{d} \Omega_{r}+\int \mathbf{L}:(\nabla \psi \otimes \mathbf{r}) \phi \mathrm{d} \Omega_{r}
\end{gathered}
$$

where $S_{r}$ is the spherical surface of radius $r \rightarrow \infty$ that delimits the chain space. According to the damage criterion, the distribution $\phi$ vanishes when $r \gg r_{\mathcal{C}}$, and thus the first term on the right-hand side becomes zero. In addition, the second term also vanishes due to the incompressibility assumption. Equation 23 can then be rewritten as

$$
\dot{\boldsymbol{\Psi}}=\int \mathbf{L}:(\nabla \psi \otimes \mathbf{r}) \phi \mathrm{d} \Omega_{r}-\int \phi_{0} \dot{\delta} \mathrm{d} \Omega_{r}
$$

By further applying the chain rule

$$
\nabla \psi=\frac{\mathrm{d} \psi}{\mathrm{d} \mathbf{r}}=\frac{\mathrm{d} \psi}{\mathrm{d} r} \frac{\mathrm{d} r}{\mathrm{~d} \mathbf{r}}=\frac{1}{r} \frac{\mathrm{d} \psi}{\mathrm{d} r} \mathbf{r}
$$


and move the velocity gradient $\mathbf{L}$ out of the integral, we finally obtain

$$
\dot{\boldsymbol{\Psi}}=\int \phi(\mathbf{t} \otimes \mathbf{r}) \mathrm{d} \Omega_{r}: \mathbf{L}-\int \phi_{0} \dot{\delta} \psi \mathrm{d} \Omega_{r}
$$

where $\mathbf{t}=(1 / r)(\mathrm{d} \psi / \mathrm{d} r) \mathbf{r}$ is the chain force. Similarly, the term corresponding to the stressfree configuration in eq 21 can be obtained as

$$
\dot{\boldsymbol{\Psi}}_{0}=\int \boldsymbol{\Phi}(\mathbf{t} \otimes \mathbf{r}) \mathrm{d} \Omega_{r}: \mathbf{L}-\int \boldsymbol{\Phi}_{0} \dot{\Delta} \psi \mathrm{d} \Omega_{r}
$$

Plugging $27 \&(28)$ in 21 , the difference $\Delta \dot{\Psi}$ becomes

$$
\begin{aligned}
\Delta \dot{\boldsymbol{\Psi}}= & {\left[\int(\phi-\boldsymbol{\Phi})(\mathbf{t} \otimes \mathbf{r}) \mathrm{d} \Omega_{r}+p \mathbf{I}\right]: \mathbf{L} } \\
& -\int\left(\phi_{0} \dot{\delta}-\boldsymbol{\Phi}_{0} \dot{\Delta}\right) \psi \mathrm{d} \Omega_{r}
\end{aligned}
$$

\section{A.3.: Parametric Study on the Damage Function}

Here we investigate how the material response depends on the critical end-to-end distance $r_{c}$ and the standard deviation $\sigma_{d}$. To illustrate this, we consider the uniaxial tension of a cylindrical sample composed of a single network that experiences a maximum stretch of $\lambda=$ 4.8. Figure 11 shows the effect of critical end-to-end distance $r_{c}$ for rupture on the mechanical response of the polymer, where we clearly see that the decrease in $r_{c}$ results in a more significant damage in the material. Interestingly, although a network with $r_{c}=0.9 \mathrm{Nb}$ experiences the least cumulative damage, as shown in Figure 11a, its stretch-stress relationship exhibits the largest hysteresis loop, indicating that the energy dissipation is highest. This is because the chains break at a higher level of stretching for large $r_{c}$. Because of the stiffening of the chains, the energy dissipated by chain breakage is higher and thus leads to a larger hysteresis loop. Therefore, the network with larger $\lambda_{c}$ is superior in both stiffness and toughness.

Figure 12 shows the effect of the standard deviation $\sigma_{d}$. In this study, the critical end-to-end distance is set to $r_{c}=0.7 \mathrm{Nb}$ and we explore 3 distint values of $\sigma_{d}\left(0.05 r_{c}, 0.15 r_{c}\right.$ and $\left.0.3 r_{c}\right)$. We observe a slight difference in the evolution of $D$ between the three networks, indicating that $\sigma_{d}$ plays an insignificant role in the cumulative damage. In terms of the mechanical response, we see that when the deformation is relatively small $(\lambda<2)$, all three materials exhibit the same stiffness. At large deformation $(\lambda>2)$, stress stiffening can be observed for the material with large $\sigma_{d}$. We note that for small $\sigma_{d}$, chain rupture occurs within a narrow range around $r_{c}$. However, this range becomes wider as $\sigma_{d}$ increases, allowing some chains to be highly stretched and stiffen before breaking. As a result, the network exhibits a higher stress and energy dissipation. 


\section{REFERENCES}

(1). Akalp U; Bryant SJ; Vernerey FJ Tuning tissue growth with scaffold degradation in enzymesensitive hydrogels: a mathematical model. Soft Matter 2016, 12 (36), 7505-7520. [PubMed: 27548744]

(2). Bacca M; Creton C; McMeeking RM A model for the mullins effect in multinetwork elastomers. J. Appl. Mech 2017, 84 (12), 121009.

(3). Canadell J; Goossens H; Klumperman B Self-healing materials based on disulfide links. Macromolecules 2011, 44 (8), 2536-2541.

(4). Chapra SC; Canale RP Numerical Methods for Engineers;. McGraw-Hill: New York, 1998; Vol. 2.

(5). Chen B-K; Wu T-Y; Chang Y-M; Chen AF Ductile polylactic acid prepared with ionic liquids. Chem. Eng. J 2013, 215-216, 886-893.

(6). de Gennes PG; Leger L Dynamics of Entangled Polymer Chains. Annu. Rev. Phys. Chem 1982, 33 (1), 49-61.

(7). De Tommasi D; Marzano S; Puglisi G; Zurlo G Damage and healing effects in rubber-like balloons. Int. J. Solids Struct 2009, 46 (22-23), 3999-4005.

(8). Dhote V; Vernerey FJ Mathematical model of the role of degradation on matrix development in hydrogel scaffold. Biomech. Model. Mechanobiol 2014, 13 (1), 167-183. [PubMed: 23636471]

(9). Diani J; Brieu M; Vacherand JM A damage directional constitutive model for mullins effect with permanent set and induced anisotropy. European Journal of Mechanics-A/Solids 2006, 25 (3), 483-496.

(10). Diani J; Fayolle B; Gilormini P A review on the mullins effect. Eur. Polym. J 2009, 45 (3), 601612.

(11). Ducrot E; Chen Y; Bulters M; Sijbesma RP; Creton C Toughening elastomers with sacrificial bonds and watching them break. Science 2014, 344 (6180), 186-189. [PubMed: 24723609]

(12). Flory PJ; Rehner J Statistical Mechanics of CrossLinked Polymer Networks I. Rubberlike Elasticity. J. Chem. Phys 1943, 11 (11), 512-520.

(13). Gong JP Why are double network hydrogels so tough? Soft Matter 2010, 6 (12), 2583-2590.

(14). Gong JP; Katsuyama Y; Kurokawa T; Osada Y Double-network hydrogels with extremely high mechanical strength. Adv. Mater 2003, 15 (14), 1155-1158.

(15). Guo Z; Sluys LJ Computational modelling of the stress-softening phenomenon of rubber-like materials under cyclic loading. European Journal of Mechanics-A/Solids 2006, 25 (6), 877-896.

(16). Harwood JAC; Mullins L; Payne AR Stress softening in natural rubber vulcanizates. part ii. stress softening effects in pure gum and filler loaded rubbers. J. Appl. Polym. Sci 1965, 9 (9), 30113021.

(17). Hutchinson JW; Neale KW Neck propagation. J. Mech. Phys. Solids 1983, 31 (5), 405-426.

(18). Kaliske M; Nasdala L; Rothert H On damage modelling for elastic and viscoelastic materials at large strain. Comput. Struct 2001, 79 (22-25), 2133-2141.

(19). Knauss WG A review of fracture in viscoelastic materials. Int. J. Fract 2015, 196 (1-2), 99-146.

(20). Kuhn W; Grun F Statistical behavior of the single chain molecule and its relation to the statistical behavior of assemblies consisting of many chain molecules. J. Polym. Sci 1946, 1 (3), 183-199.

(21). Lake GJ; Thomas AG The strength of highly elastic materials. Proc. R. Soc. London, Ser. A 1967, 300 (1460), 108-119.

(22). Lavoie SR; Long R; Tang T A rate-dependent damage model for elastomers at large strain. Extreme Mechanics Letters 2016, 8, 114-124.

(23). Li J; Mayau D; Lagarrigue V A constitutive model dealing with damage due to cavity growth and the mullins effect in rubber-like materials under triaxial loading. J. Mech. Phys. Solids 2008, 56 (3), 953-973.

(24). Lin W-C; Fan W; Marcellan A; Hourdet D; Creton C Large strain and fracture properties of poly (dimethylacrylamide)/silica hybrid hydrogels. Macromolecules 2010, 43 (5), 2554-2563.

(25). Long R; Hui C-Y Crack tip fields in soft elastic solids subjected to large quasi-static deformationa review. Extreme Mechanics Letters 2015, 4, 131-155. 
(26). Mao Y; Lin S; Zhao X; Anand L A large deformation viscoelastic model for double-network hydrogels. J. Mech. Phys. Solids 2017, 100, 103-130.

(27). Mao Y; Talamini B; Anand L Rupture of polymers by chain scission. Extreme Mechanics Letters 2017, 13, 17-24.

(28). Marckmann G; Verron E; Gornet L; Chagnon G; Charrier P; Fort P A theory of network alteration for the Mullins effect. J. Mech. Phys. Solids 2002, 50 (9), 2011-2028.

(29). Merckel Y; Brieu M; Diani J; Caillard J A mullins softening criterion for general loading conditions. J. Mech. Phys. Solids 2012, 60 (7), 1257-1264.

(30). Millereau PM Large Strain and Fracture of Multiple Network Elastomers. PhD Thesis, Paris 6, 2017.

(31). Na Y-H; Kurokawa T; Katsuyama Y; Tsukeshiba H; Gong JP; Osada Y; Okabe S; Karino T; Shibayama M Structural characteristics of double network gels with extremely high mechanical strength. Macromolecules 2004, 37 (14), 5370-5374.

(32). Na Y-H; Tanaka Y; Kawauchi Y; Furukawa H; Sumiyoshi T; Gong JP; Osada Y Necking phenomenon of double-network gels. Macromolecules 2006, 39 (14), 4641-4645.

(33). Nakajima T; Furukawa H; Gong JP; Lin EK; Wu W-1. A deformation mechanism for doublenetwork hydrogels with enhanced toughness. Macromol. Symp 2010, 291-292, 122-126.

(34). Ogden RW; Roxburgh DG A pseudo-elastic model for the mullins effect in filled rubber. In Proceedings of the Royal Society of London A: Mathematical. Proc. R. Soc. London, Ser. A 1999, 455, 2861-2877.

(35). Podsiadlo P; Qin M; Cuddihy M; Zhu J; Critchley K; Kheng E; Kaushik AK; Qi Y; Kim H.-Sug; Noh Si-Tae; et al. Highly ductile multilayered films by layer-by-layer assembly of oppositely charged polyurethanes for biomedical applications. Langmuir 2009, 25 (24), 14093-14099. [PubMed: 19824626]

(36). Qi HJ; Boyce MC Constitutive model for stretch-induced softening of the stress-stretch behavior of elastomeric materials. J. Mech. Phys. Solids 2004, 52 (10), 2187-2205.

(37). Shen T; Garriga Font M; Jung S; Gabriel ML; Stoykovich MP; Vernerey FJ Remotely triggered locomotion of hydrogel magbots in confined spaces. Sci. Rep 2017, 7 (1), 16178. [PubMed: 29170417]

(38). Simo JC On a fully three-dimensional finite-strain viscoelastic damage model: formulation and computational aspects. Computer methods in applied mechanics and engineering 1987, 60 (2), 153-173.

(39). Sridhar SL; Schneider MC; Chu S; de Roucy G; Bryant SJ; Vernerey FJ Heterogeneity is key to hydrogel-based cartilage tissue regeneration. Soft Matter 2017, 13 (28), 4841-4855. [PubMed: 28613313]

(40). Sun J-Y; Zhao X; Illeperuma WRK; Chaudhuri O; Oh KH; Mooney DJ; Vlassak JJ; Suo Z Highly stretchable and tough hydrogels. Nature 2012, 489 (7414), 133. [PubMed: 22955625]

(41). Talamini B; Mao Y; Anand L Progressive damage and rupture in polymers. J. Mech. Phys. Solids 2018, 111, 434-457.

(42). Thomas AG Rupture of rubber. ii. the strain concentration at an incision. J. Polym. Sci 1955, 18 (88), 177-188.

(43). Treloar LRG The elasticity of a network of long-chain molecules. I. Trans. Faraday Soc 1943, 39 (0), 36-41.

(44). Treloar LRG The Physics of Rubber Elasticity; Oxford University Press: 1975.

(45). Vernerey FJ; Long R; Brighenti R A statistically-based continuum theory for polymers with transient networks. J. Mech. Phys. Solids 2017, 107, 1-20.

(46). Vernerey F; Liu WK; Moran B Multi-scale micromorphic theory for hierarchical materials. J. Mech. Phys. Solids 2007, 55 (12), 2603-2651.

(47). Vernerey F; Shen T The mechanics of hydrogel crawlers in confined environment. J. R. Soc., Interface 2017, 14 (132), 20170242. [PubMed: 28747396]

(48). Vernerey FJ Transient response of nonlinear polymer networks: A kinetic theory. J. Mech. Phys. Solids 2018, 115, 230-247. 
(49). Volokh KY On modeling failure of rubber-like materials. Mech. Res. Commun 2010, 37 (8), 684-689.

(50). Volokh KY Review of the energy limiters approach to modeling failure of rubber. Rubber Chem. Technol 2013, 86 (3), 470-487.

(51). Wang X; Hong W Pseudo-elasticity of a double network gel. Soft Matter 2011, 7 (18), 85768581.

(52). Webber RE; Creton C; Brown HR; Gong JP Large strain hysteresis and mullins effect of tough double-network hydrogels. Macromolecules 2007, 40 (8), 2919-2927.

(53). White ZW; Vernerey FJ Armours for soft bodies: How far can bioinspiration take us? Bioinspir. Biomim 2018, 13, 041004. [PubMed: 29595522]

(54). Wineman A Nonlinear Viscoelastic Solids:A Review. Mathematics and Mechanics of Solids 2009, 14 (3), 300-366.

(55). Wineman A Time dependent void growth in elastomers undergoing chemo-mechanical evolution. Journal of Elasticity 2015, 121 (2), 255-274.

(56). Wirthl D; Pichler R; Drack M; Kettlguber G; Moser R; Gerstmayr R; Hartmann F; Bradt E; Kaltseis R; Siket CM; et al. Instant tough bonding of hydrogels for soft machines and electronics. Sci. Adv 2017, 3 (6), e1700053. [PubMed: 28691092]

(57). Zhao X A theory for large deformation and damage of interpenetrating polymer networks. J. Mech. Phys. Solids 2012, 60 (2), 319-332. 


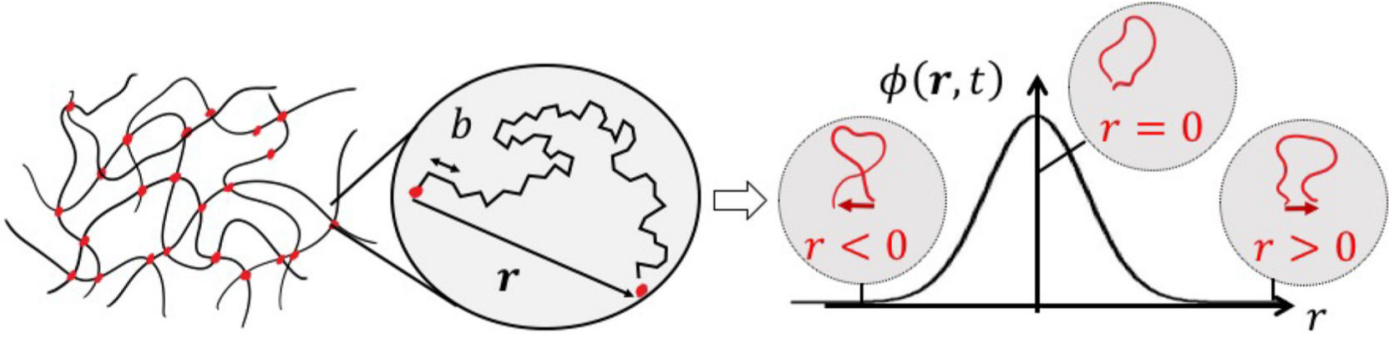

(a) Polymer network and chain's end-to-end vector

(b) Distribution of end-to-end vector

Figure 1.

(a) Cross-linked polymer network and illustration of the end-to-end vector $\mathbf{r}$ of a single chain. The chain is assumed to consist of $N$ Kuhn segments with length $b$. (b) Statistically, the chain population can be described by the distribution $\phi(\mathbf{r}, t)$, for which we show an example in one dimension. 


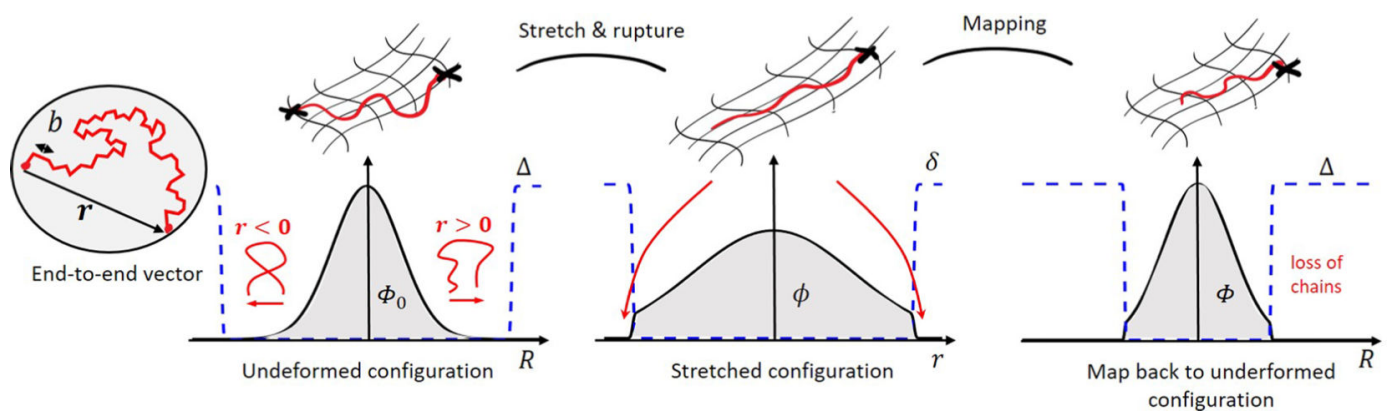

Figure 2.

Illustration of the evolution equation for the chain distribution. Deformation has the effect of stretching the distribution without affecting its overall area; chain rupture at higher stretch tends to decrease the number of chain with a large end-to-end distance. 


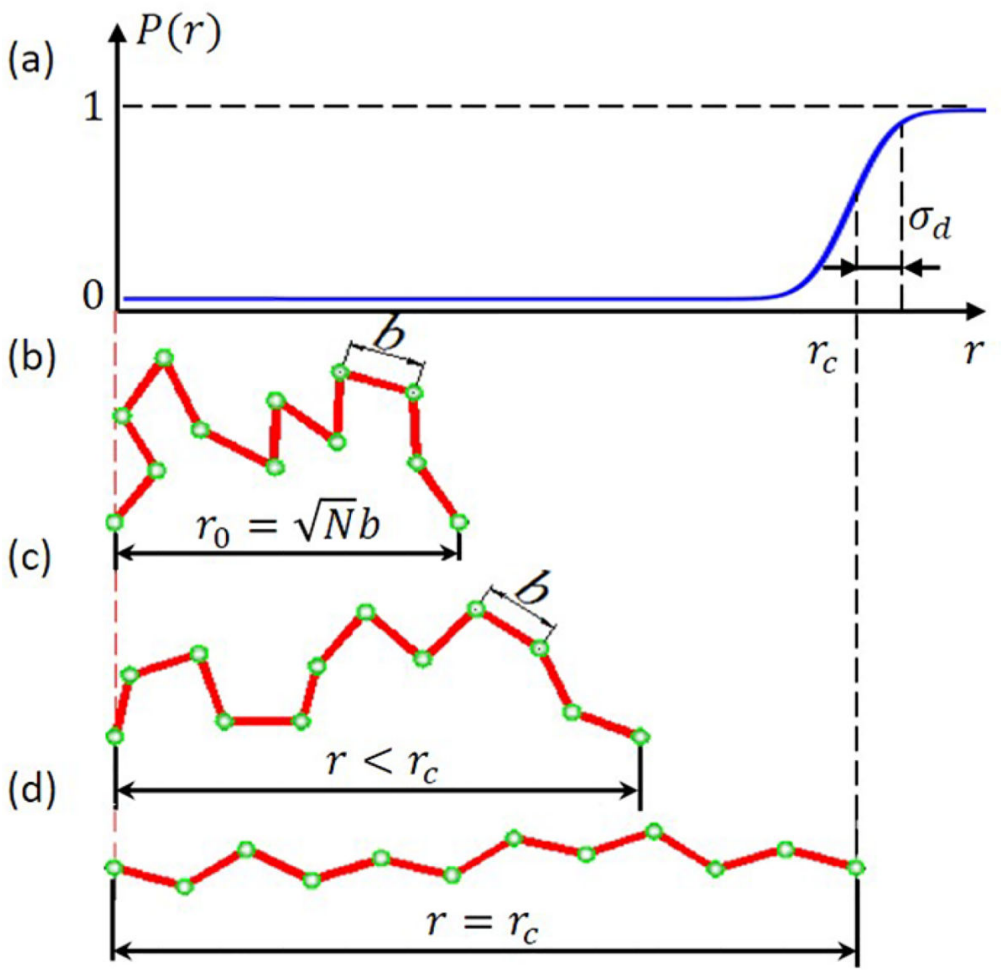

Figure 3.

(a) Probability of chain damage $P$ as a function of the end to-end distance $r$. (b-d) Elongation of a chain from its natural state to the critical end-to-end distance for failure $r_{\mathrm{c}}$ 
(a)

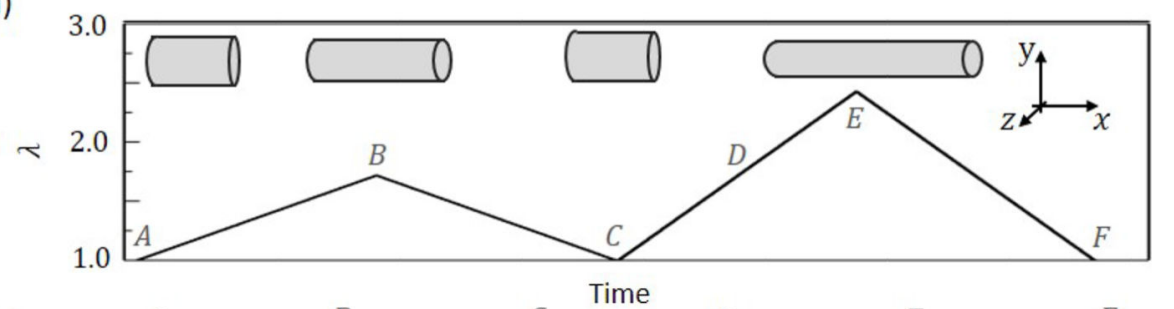

(b)
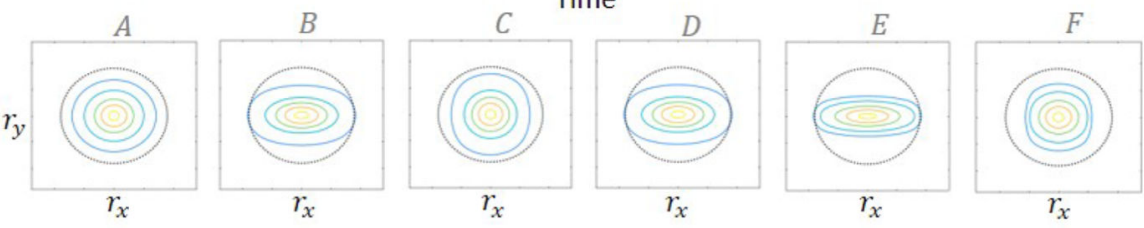

(c)

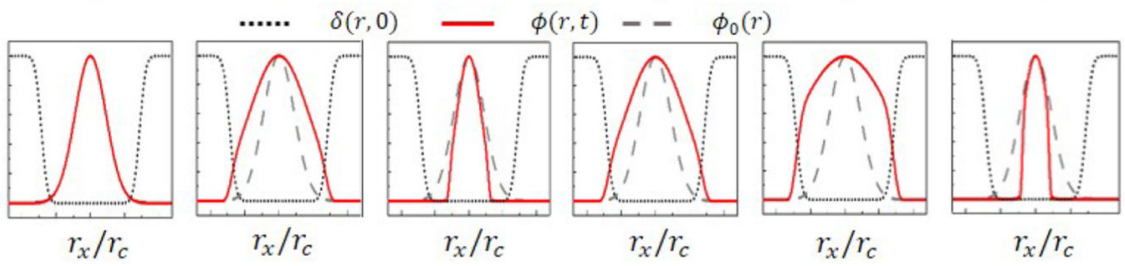

(d)

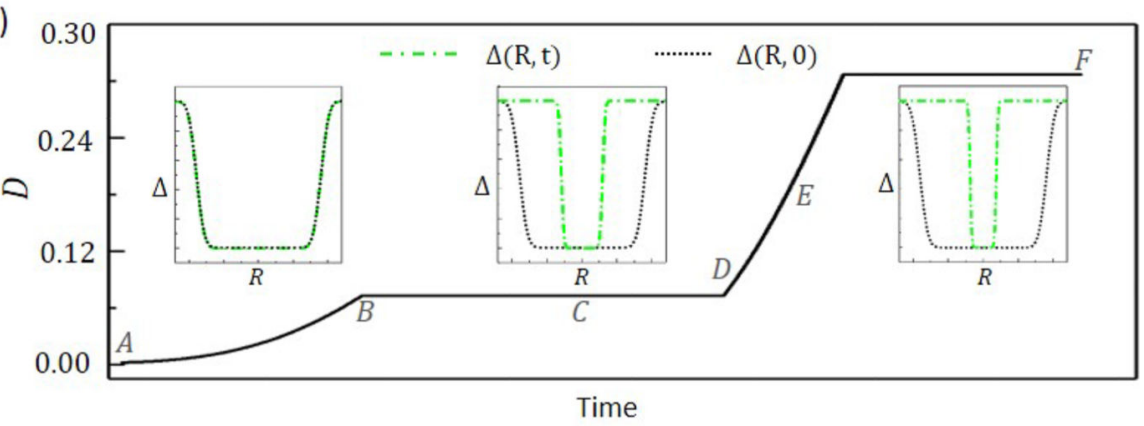

Figure 4.

(a) Loading history of the material. (b) The subfigures about the cross section of the distribution at the $x-z$ and (c) their corresponding 1-D projection along the $x$-direction. (d) Evolution of chain damage $D$ in time and the evolution of $\Phi(\mathrm{R}, t)$ during the deformation history. 
(a)

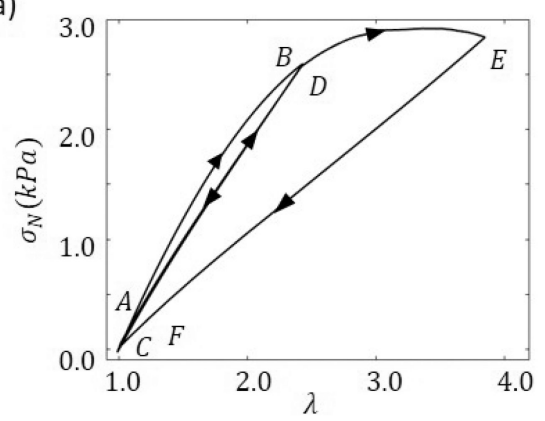

(b)

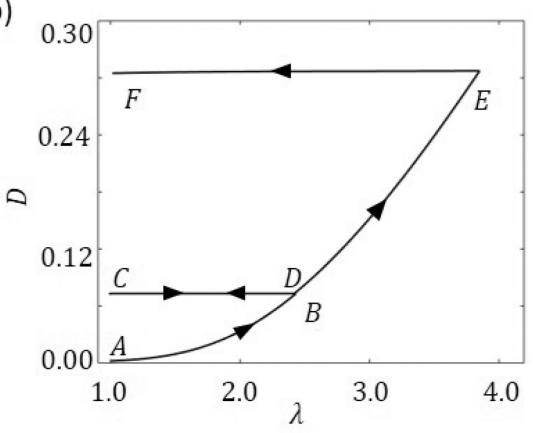

Figure 5.

(a) Stress-strain relationship and (b) the damage-strain relationship over the course of deformation. 
(a)
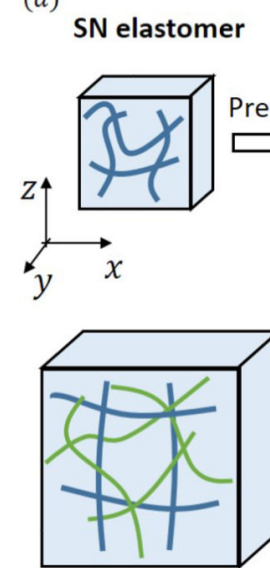

(b)
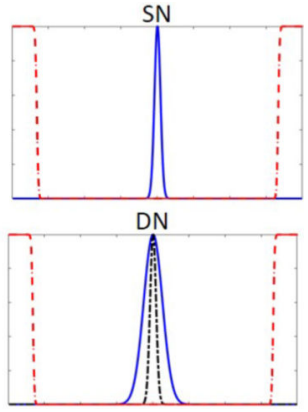

TN elastomer
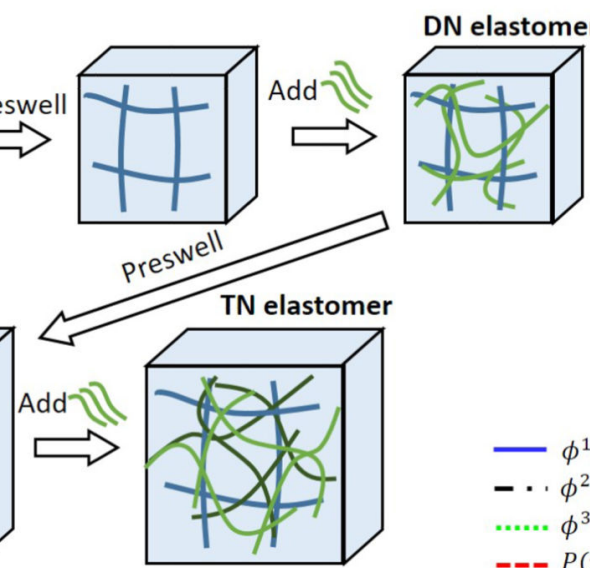
(1)
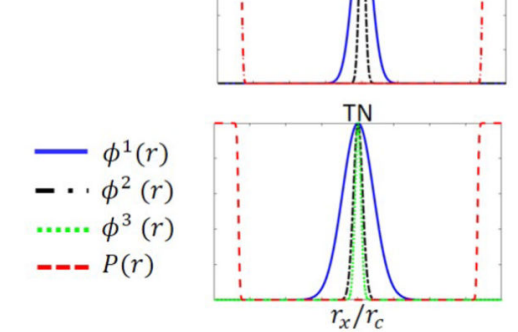

Figure 6.

(a) Schematic of experimental synthesis of DN and TN elastomers. (b) 1-D distribution of $\mathrm{SN}, \mathrm{DN}$, and TN elastomers at the initial state. 
(a)

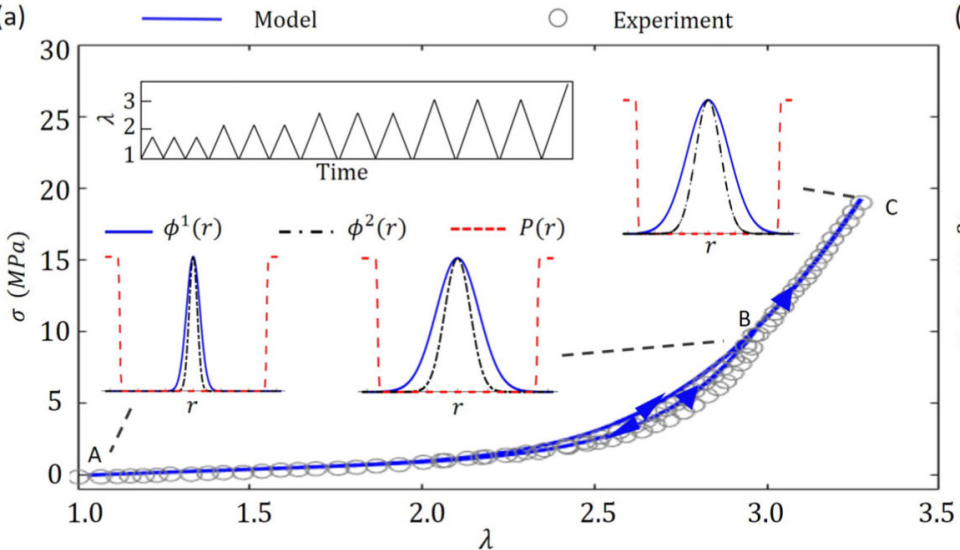

(b)

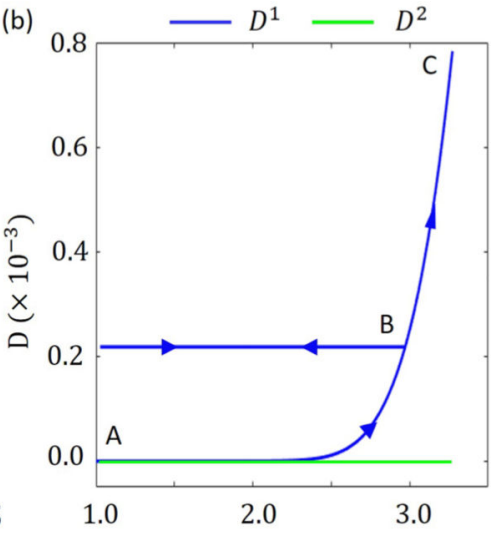

Figure 7.

(a) Matching between model prediction and the experimental measurement of the forcedeformation relationship for the DN elastomer. The subfigures show the evolution of chain distribution of each network. (b) Damage-deformation relationship of each network. 
(a)

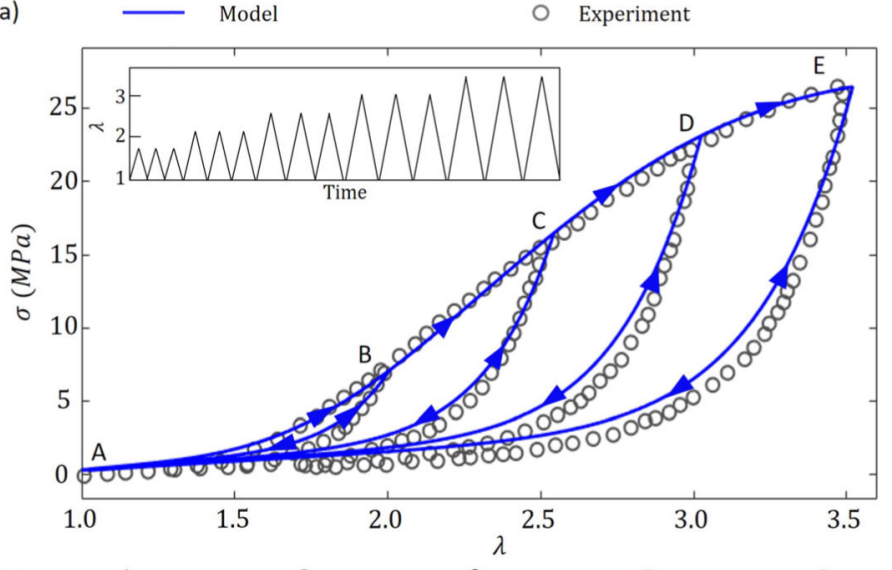

(c)

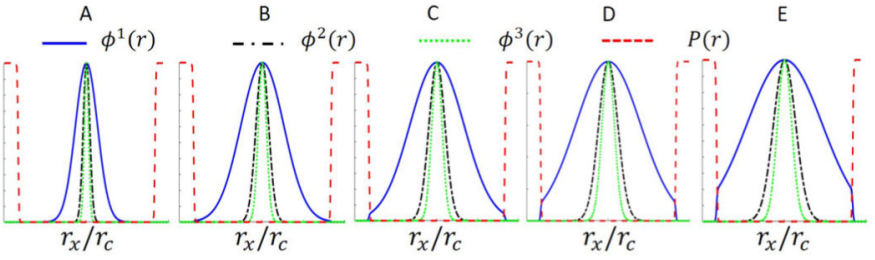

(b) $-\sigma^{1} \quad \cdots \cdot \sigma^{2} \quad \cdots \cdots \cdots \cdot \sigma^{3}$

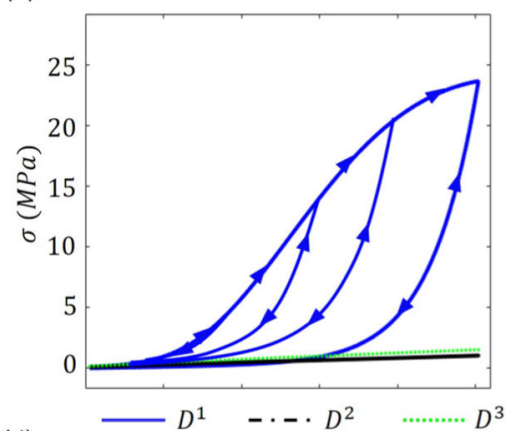

(d)

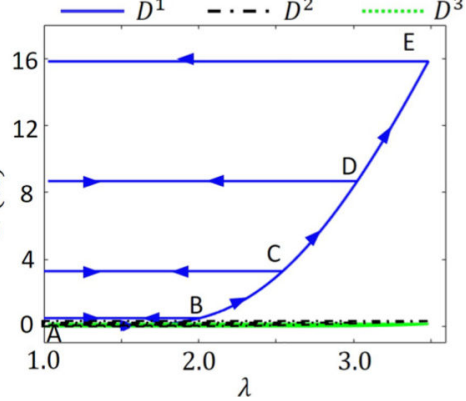

Figure 8.

(a) Matching between model prediction and the experimental measurement of the forcedeformation relationship for the TN elastomer. (b) Stress-deformation relationship of each individual network. (c) Five snapshots that show the evolution of distribution $\phi(r)$ during the loading. (d) Damage-deformation relationship of each network. 
(a)

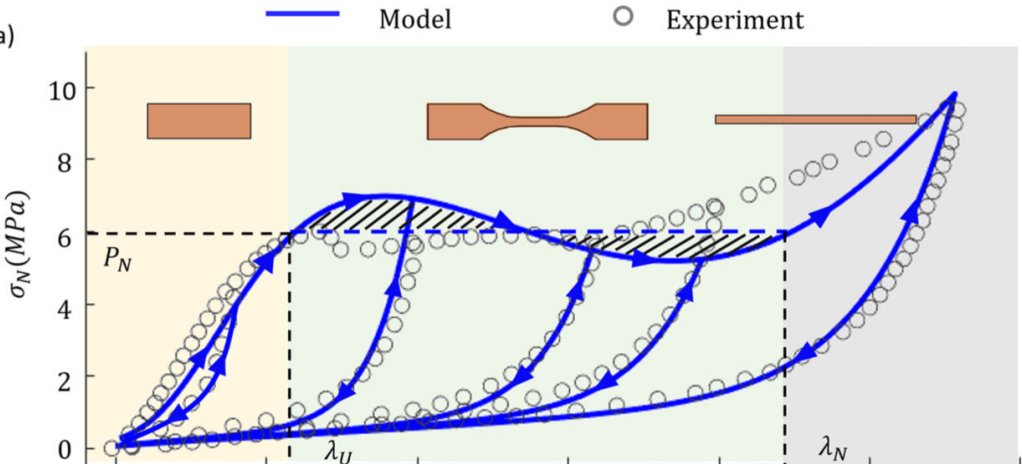

(b)

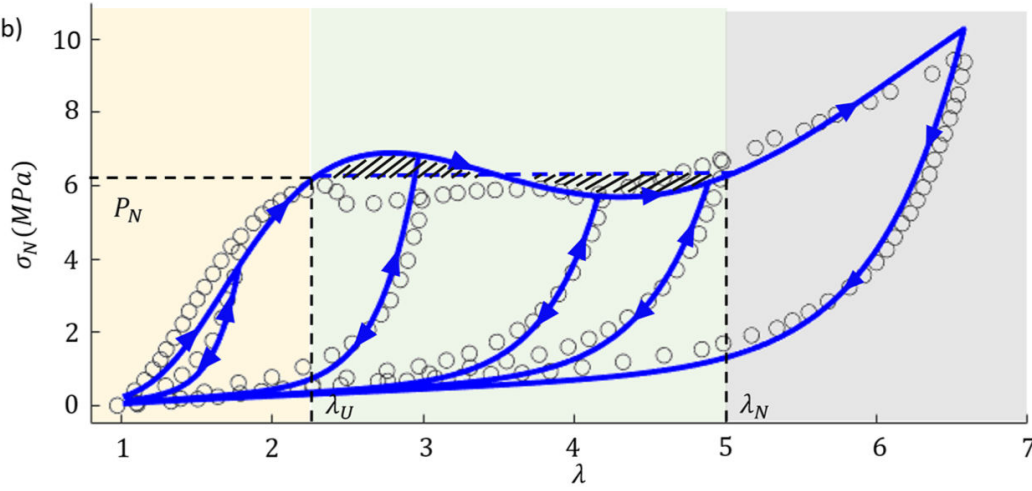

(c) $-1^{\text {st }}-\cdot 2^{\text {nd }} \cdots 3^{r d}-4^{\text {th }}$

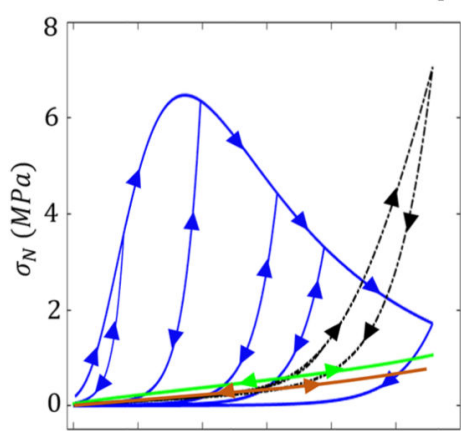

(d)

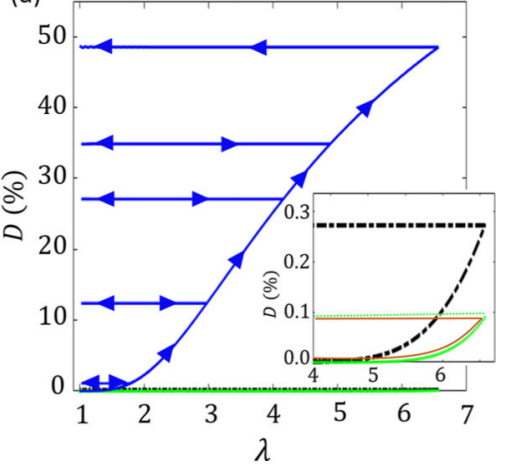

Figure 9.

Matching between model prediction and the experimental measurement of the stress-stretch relationship for the QN elastomer. For (a), we used the parameters calibrated in section 4.1, and in (b), we slightly modified the Kuhn segment number of the second network as $\mathrm{N}_{2}=$ 170. (c) Stress-deformation relationship of each individual network. (d) Damagedeformation relationship of each network. 
(a)

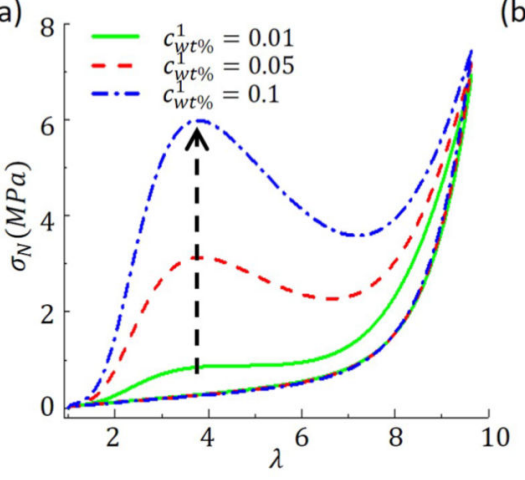

(b)

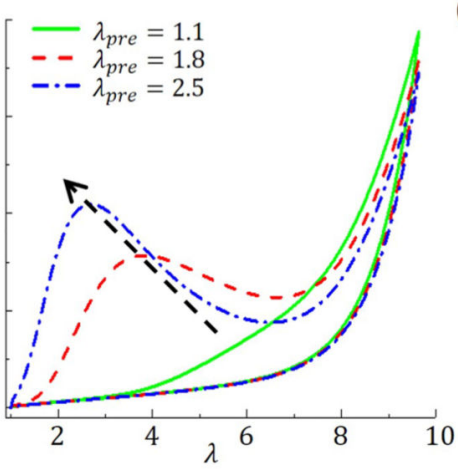

(c)

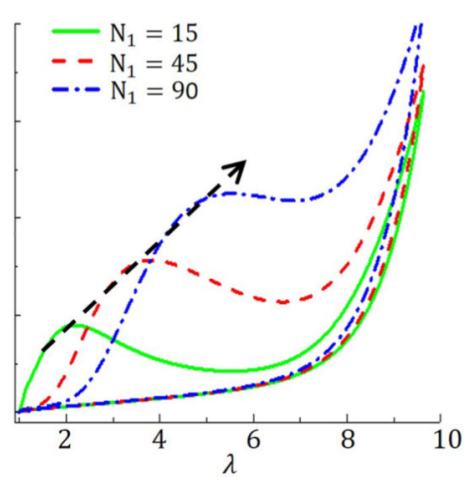

Figure 10.

Effect of (a) $c_{\text {wt } \%}^{1}$, (b) $\lambda_{\text {pre }}$, and (c) $N_{1}$ on necking phenomena of hydrogels during stretching. The parameters used are $c_{\mathrm{wt} \%}^{1}=0.05, \lambda_{\mathrm{pre}}=1.8, N_{1}=45$, and $N_{2}=170$ if not specified otherwise. 

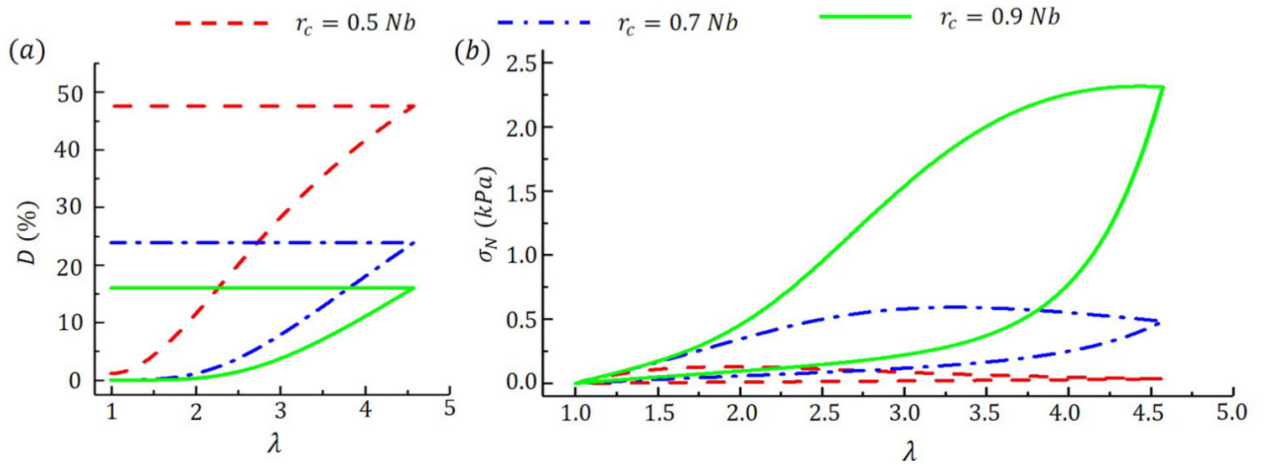

Figure 11.

Effect of critical end-to-end distance $r_{\mathcal{c}}$ on the (a) the cumulative chain damage and (b) the mechanical response of a single elastic network during the course of a uniaxial tension test. 

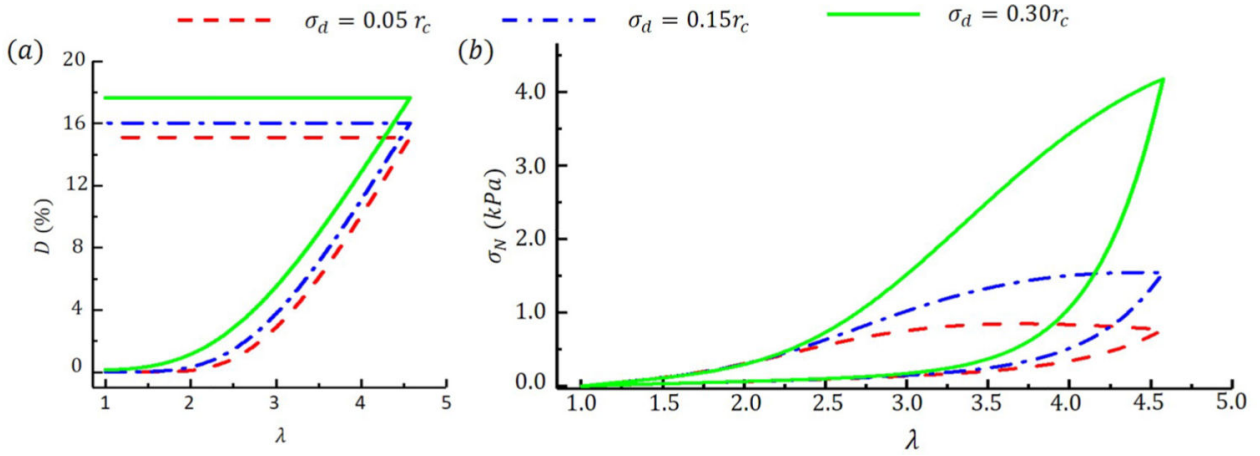

Figure 12.

Effect of standard deviation of chain damage $\sigma_{d}$ on the (a) the cumulative chain damage and (b) the mechanical response of a single elastic network during the course of a uniaxial tension test. 